(prednisolone) and propranolol are commonly prescribed and lesions unresponsive (15\% cases) have been treated with antiangiogenic agents such as IFN-alpha, sirolimus, vincristine and cyclophosphamide.Invasive measures like hepatic artery ligation or embolization may be tried in complicated cases. Surgical therapy is recommended for uni-lobe lesions, masses with low potential for regression or in suspected malignancy.

In summary, this case always had a poor prognosis as it was multifocal, had intra-hepatic shunting and was complicated by CCF and haemorrhage. Unfortunately TAE wasn't successful on this occasion but sub-speciality involvement was greatly appreciated.

\section{P458 ARE PATENT DUCTUS ARTERIOSUS PAINFUL ENOUGH TO BE TREATED WITH PARACETAMOL?}

Anna Katherine Darbyshire*, Isha Nijjar, Shree Vishna Rasiah. Birmingham Women's and Children's NHS Foundation Trust, Birmingham, UK

\subsection{6/archdischild-2019-epa.794}

Background Patent ductus arteriosus causes significant dilemma about its management in the neonatal units around the world today. Historically, cyclooxygenase (COX inhibitors) such as Indomethacin or Ibuprofen have been shown to be effective pharmacological treatments for closing a PDA. Ibuprofen is currently the main pharmacotherapy in neonatal units in the UK since Indomethacin is no longer available. More recently, paracetamol has been suggested as an alternative to ibuprofen, though there is a paucity of evidence for its use. We started using paracetamol for the closure of PDA in preterm babies where there were contraindications to Ibuprofen.

Aim To determine the frequency of paracetamol use for the treatment of patent ductus arteriosus and the outcomes for the babies.

Methods We retrospectively reviewed the BadgerNet to identify all babies under 28 weeks gestation who received paracetamol for treatment of patent ductus arteriosus between $1^{\text {st }}$ January 2017 to $31^{\text {st }}$ December 2018. The notes were reviewed in order to ascertain the reason for paracetamol, as opposed to ibuprofen, as well as the rate of PDA ligation and final outcomes of the babies.

Results In the last two years, 21 babies were treated with paracetamol for their patent ductus arteriosus. The average gestation of babies receiving paracetamol was 24 completed weeks and the average weight was $710 \mathrm{~g}$. Paracetamol was given between day 2 and day 29, but on average it was given on day 11 of life. In all cases, paracetamol was used because ibuprofen was contraindicated. The main reason for contraindication was low platelets in 10 babies and 5 with evolving or intraventricular haemorrhage. Three babies were because of renal failure, two with abdominal concerns and one with a pulmonary haemorrhage. Six babies went on to have PDA ligations performed following paracetamol treatment. In terms of outcomes, $71 \%$ survived and 29\% died.

Conclusions In our practice, paracetamol has been used to treat patent ductus arteriosus in babies where Ibuprofen was contraindicated. This was for ventilated babies less than 28 weeks gestation. Nearly $30 \%$ of babies still required PDA ligation despite treatment with paracetamol. We believe further research is required in the use of paracetamol for the treatment of PDA. Where there are contraindications to Ibuprofen, paracetamol can be used cautiously to treat PDA.
P459 LOOKING AT THE LIPIDS: QUALITY IMPROVEMENT IN THE NICU

Carmel Maria Moore*, Peter Duddy, Anne Doolan, Jan Miletin. Coombe Women and Infants University Hospital, Dublin 8, Ireland

\subsection{6/archdischild-2019-epa.795}

Background and aims Parenteral nutrition is a vital part of our management of neonates in the NICU. The National Paediatric and Neonatal Parenteral Nutrition Guidelines were introduced in November 2016. The national guidelines recommend checking serum triglyceride levels on day 3-4 and day 5-7 of $\mathrm{PN}$ administration, as well as weekly thereafter if stable. We noted that this was not occurring in our unit and so aimed to audit compliance with national guidelines on lipid monitoring in neonates receiving parenteral nutrition in order to target a quality improvement initiative.

Methods This was a retrospective review of all babies on PN for greater than one week. Cases were identified using the PN order database maintained by the pharmacy team. The laboratory system was interrogated to assess if the babies had triglycerides or lipid indexes checked.

Results 20 babies on PN for greater than one week over the past six months. Only $15 \%$ of babies $(n=3)$ had any lipid monitoring performed and no babies had been monitored in compliance with the guidelines. Some babies had both lipid index (performed on site) and triglycerides (performed off site, with a varying turnaround time.

Conclusion There is significant room for improvement in our management of lipid monitoring in our NICU and we are going to initiate a quality improvement initiative to improve compliance with the guidelines including education sessions with NICU staff.

\section{P460 AN AUDIT ON MANAGMENT OF PAIN IN PRETERM BABIES IN UNIVERSITY MATERNITY HOSPITAL LIMERICK}

Muhammad Zia*, Aisha ljaz, Raef Abouelhussein, Niazy Alasaaf, Conn Sreenan, Rizwan Khan. University Maternity Hospital Limerick, limerick, Ireland

10.1136/archdischild-2019-epa.796

Background Pre term babies admitted in ICU settings routinely undergo many painful procedures influencing their long term development. We can improve the quality of care for these babies by following simple measures that are cost effective,safe and less time consuming.

Aim of the audit The aim of the audit was to look for pharmacological and non pharmacological measures being followed to reduce acute and procedural pain in pre term babies admitted in neonatal intensive care unit of University Maternity Hospital,Limerick.

Methodology Audit was prospective.

We followed 20 pre term babies who were admitted in NICU during 15 December 2018 to 31 January,2109.An already designed proforma was used to collect data regarding prematurity,name of procedure(heelstick,IV cannulation,phlebotomy,NG insertion,tracheal intubation, ROP screening,LP) and method of pain relief used.Data was analysed on the basis of pharmacological(sucrose,paracetamol) and non pharmacological measures(swaddling,non nutritive sucking,positioning,skin to skin care, breast feeding).

Results

1. Gestational age varies from $28+0$ to $34+2$ weeks. 